\title{
Visualizing the unseen
}

\author{
Scientific illustrations help to communicate complex information and data across disciplines. The work of science \\ animators and illustrators is therefore a valuable part of effective science communication, in particular for \\ microbiology, as the majority of organisms studied cannot be seen with the naked eye.
}

F rom the moment we open our eyes in the morning to the time we go to sleep, visual stimuli guide us throughout the day. We watch the news, we see street signs and we read messages on our phones. A lot of what we see involves illustrations, photographs and videos that inform us about a specific topic. Visual information makes use of colour, shape, space and even movement to convey a message. For some, these visual materials help in the formation of memories, since the cognitive process is usually more efficient when processing static or moving visual information as compared to plain text.

This is particularly relevant when it comes to science. Areas that focus on the study of the micro-, nano- or picometre world, in which structures and organisms are invisible to the naked eye, rely on images to convey findings and messages. Microbiology and molecular and cellular biology are two examples. We can read about the composition of a virus, a protein's intricate structure or the process of DNA replication, but without a visual representation it is difficult to fully comprehend these complex systems. Fortunately, thanks to the advance of technology, scientists can use a variety of tools to help visualize how microscopic elements are composed and how they function. Fluorescence microscopy can allow us to see, for example, a bacterium infecting a mammalian cell, while electron microscopy can generate high-resolution images of a cell's ultrastructure. To get deeper insights into the atomic structure of molecules and cells, complex techniques like X-ray diffraction, nuclear magnetic resonance or cryo-electron microscopy can be used. These techniques continue to provide unprecedented insights into what microbes and their components look like, yet they require specific expertise and are still somewhat restricted to a small audience, particularly since such images can be relatively ineffective in communicating information to non-experts and the general public.

That's where scientific animation and illustration come in. These disciplines gather complex and specialized scientific information and combine it with more abstract concepts like artistic beauty and composition. This translates into an aesthetically engaging and broadly understandable figure.

Art and science, in particular microbiology, have long gone hand in hand. An article by Frédéric Barras (Barras, F. Environ. Microbiol. Rep. 11, 29-34 (2019)) summarized the connection between microbiology and art throughout history. More recently, Graham Johnson's animation from 1999 shows how the motor protein kinesin walks along a protofilament of a microtubule, Michele Banks creates watercolour collages and Petri dish paintings using an interesting artistic process (Jermy, A. Nat Microbiol. 1, 15013 (2016)) involving medieval novels about the plague and experimentation with colour palettes, David Goodsell has created many iconic watercolour images, and Digizyme has developed 'molecular landscape' images by integrating structural and biochemical data to show the intricate complexity of living cells, which are just a few examples. These visualizations are particularly effective for non-specialized audiences. They provide a gateway into the cellular world, allowing something that seemed unintelligible to become more tangible. The ultimate goal of animation and illustration is effective communication - to take something complex and make it accessible, visually attractive and as close to an approximation of reality as possible to capture a broad audience.

So at this point you may wonder, who are the people behind scientific animations and illustrations? Are they illustrators or are they scientists? Well, the answer is, they are both. Usually, scientific illustrators and animators are professionals who have some level of scientific knowledge complementary to their artistic design skills. They can be scientists who learnt art, or artists with a deep interest in science. In any case, they have a combined passion for both disciplines. They exhaustively research and read about the scientific topic that they want to illustrate and even work side by side with scientists to ensure accuracy, while also applying artistic license to create an illustration that is both precise and aesthetically pleasing.

Throughout history many well-known scientists have adopted illustration as a way of developing their research questions, including the Dutch microbiologist Antoni van Leeuwenhoek, who first drew 'animalcules' in the 17th century; the Spanish neuroscientist Santiago Ramon y Cajal, who created beautiful illustrations of neurons; the German biologist Ernst Haeckle, whose detailed illustrations of living creatures from the 20th century are still a source of inspiration and astonishment; and the usually overlooked Maria Sibylla Merian and other female scientists who drew illustrations in the 18th, 19 th and 20th centuries.

In this issue of Nature Microbiology, three contemporary scientific illustrators and animators share their experiences of bringing together art and science, and the different career paths available in science visualization. Biotechnologist Thom Leach tells us how he transitioned from the lab bench to the illustration studio; Janet Iwasa, a molecular biologist, assistant professor and head of The Animation Lab at the University of Utah, tells us how her animation team helps scientists to bring their hypothesis to life; and artist Beth Anderson walks us through her career as a scientific animator and her collaborations with research institutions and editorial publishers. These are just a few representative examples of the vast pool of talented scientific illustrators. Each illustrator uses a different combination of tools and resources, whether that be digital and/or traditional drawing techniques, 2D and/or 3D illustrations or animation software, and each has their own style. What they have in common is a passion and commitment to communicate science in the best possible way. Their dedication and their work should encourage scientists to improve communication to both their peers and the general public. The emergence of SARS-CoV-2 has demonstrated the value of communicating timely scientific discoveries to the general public in a clear and understandable way to avoid misunderstanding or introducing misconceptions not grounded in scientific data.

Although scientific visualization is not often prioritized in academic research, it is a key aspect of communicating new scientific discoveries to both academic and non-academic audiences. 
When done properly, images and animations allow information to be conceptually assimilated more effectively than words. Therefore, collaboration between researchers and illustrators is crucial for the effective communication of science. So it is our - and your - responsibility as scientists to make effective use of different communication routes and to commit to improving visualization at all times, but particularly when microbiology is prominently in the public eye.

Published online: 4 January 2022

https://doi.org/10.1038/s41564-021-01044-1 